\title{
Optimal Methods for Using Posterior Probabilities in Association Testing
}

\author{
Keli Liu ${ }^{a}$ Alexander Luedtke ${ }^{b}$ Nathan Tintle \\ a Department of Statistics, Harvard University, Cambridge, Mass., ${ }^{b}$ Division of Biostatistics, University of \\ California-Berkeley, Berkeley, Calif., and ' Department of Mathematics, Statistics and Computer Science, \\ Dordt College, Sioux Center, lowa, USA
}

\section{Key Words}

Imputation · Dosage · Genome-wide association studies

\begin{abstract}
Objective: The use of haplotypes to impute the genotypes of unmeasured single nucleotide variants continues to rise in popularity. Simulation results suggest that the use of the dosage as a one-dimensional summary statistic of imputation posterior probabilities may be optimal both in terms of statistical power and computational efficiency; however, little theoretical understanding is available to explain and unify these simulation results. In our analysis, we provide a theoretical foundation for the use of the dosage as a onedimensional summary statistic of genotype posterior probabilities from any technology. Methods: We analytically evaluate the dosage, mode and the more general set of all one-dimensional summary statistics of two-dimensional (three posterior probabilities that must sum to 1) genotype posterior probability vectors. Results: We prove that the dosage is an optimal one-dimensional summary statistic under a typical linear disease model and is robust to violations of this model. Simulation results confirm our theoretical findings. Conclusions: Our analysis provides a strong theoretical basis for the use of the dosage as a one-dimensional summary statistic of genotype posterior probability vectors in related tests of genetic association across a wide variety of genetic disease models.

Copyright $\odot 2013$ S. Karger AG, Basel
\end{abstract}

\section{KARGER}

E-Mail karger@karger.com

www.karger.com/hhe

\section{Introduction}

Access to high-throughput genotype data has facilitated the process of identifying the genetic component of complex diseases through genome-wide association studies. However, directly measured genotype data often only covers a fraction of known single nucleotide polymorphisms (SNPs). Increasingly, genetic analyses leverage linkage disequilibrium to impute untyped SNPs. Analysis at untyped SNPs using linkage disequilibrium information from reference panels, such as The International HapMap Project [1] or the 1000 Genomes Project [2], has already yielded promising disease loci for major depressive disorder [3], Crohn's disease [4], and prostate cancer [5] among others.

While many imputation algorithms exist (e.g. $\mathrm{MaCH}$ [6], and IMPUTE [7] among others), most algorithms generate a set of three posterior probabilities for each individual at each imputed SNP, representing the relative likelihood that the individual is actually each of the three possible genotypes at the SNP locus. While some exceptions exist (e.g. $[8,9])$, most researchers attempt to use a one-dimensional summary statistic of the two-dimensional posterior probabilities vector (three posterior probabilities that must sum to 1 ) in place of the (unknown) true genotype in downstream statistical analyses. Common choices for the one-dimensional summary statistics are the mode and the weighted mean (dosage) of 
the three posterior probabilities. While posterior probabilities are common for imputed genotypes, they also occur when using next-generation sequencing data and SNP microarray technology.

Recently, extensive simulations demonstrated that the dosage retained enough information that, in most realistic settings, the use of computationally intensive mixture models which account for the entire posterior probability vector improved power negligibly over a simpler, faster analysis using the dosage [10]. Furthermore, the dosage consistently outperformed the mode.

Despite these simulation results, little theoretical work has been conducted to consider whether the dosage will always perform optimally relative to the mode. Furthermore, while the dosage is a reasonable choice of one-dimensional summary statistic, it is unknown if more optimal summary statistics are available. In the following, we provide analytic proof that across a variety of disease models dosage will always outperform the mode. We then show that the dosage is equivalent to the optimal one-dimensional summary statistic up to a perturbation term which is essentially 0 in all practical situations. We confirm our analytic results using simulation.

\section{Methods}

The Methods section is organized as follows. First, we provide an overview of our notation and the main genetic disease model under consideration. We then provide proof that the score test using the dosage is equivalent to the score test using the entire vector of posterior probabilities. We then show that the dosage outperforms the mode and is, in fact, the optimal choice of one-dimensional summary statistic across linear genetic disease models, and is a robust choice for non-linear models. We conclude the Methods section by describing simulation analyses used to confirm the analytic results.

\section{Basic Notation and Disease Model}

Individual genotypes provided by imputation software, as well as some SNP microarray and sequencing technology, are typically provided for each individual $i$ as a vector of three posterior probabilities $\alpha_{i} \triangleq\left(\alpha_{i 0}, \alpha_{i 1}, \alpha_{i 2}\right)$, where $\alpha_{i k}$ is the posterior probability that individual $i$ has $k$ minor alleles, $k=0,1,2$ at a SNP of interest. The vector of posterior probabilities $\alpha_{i}$ can be interpreted as suggesting that the true minor allele count for individual $i$, denoted $x_{i}$, can be modeled as being a single random draw from a multinomial distribution with probabilities indicated by $\alpha_{i}$. We assume that $\alpha_{i}$ is known for each individual. Let $y_{i}$ be an indicator for disease status and let the probability of disease for individual $i$ be given as $\pi_{i}\left(x_{i}\right)$. A general formulation for the disease-genotype relationship is $y_{i} \mid x_{i} \sim \operatorname{Bern}\left(\pi_{i}\left(x_{i}\right)\right)$, where $\pi_{i}=f\left(x_{i}, \beta\right)$. We assume that $f$ is a smooth function. and $\beta$ is a parameter vector constrained so that the range of $f$ is some subset of the unit interval. We will use the term addi- tive model to mean that function $f$ depends on $x_{i}$ and $\beta$ only through a term of the form $\beta_{0}+\beta_{1} x_{i}$. In this paper, we explore 2 types of additive models: (1) a linear additive model: $\pi_{i}=\beta_{0}+\beta_{1} x_{i}$, and (2) a (non-linear) logistic additive model,

$$
\pi_{i}=\frac{e^{\beta_{0}+\beta_{1} x_{i}}}{1+e^{\beta_{0}+\beta_{1} x_{i}}} .
$$

When $x_{i}$ is unobserved, it is common to naively plug in a onedimensional summary statistic such as the mode $m_{i} \triangleq \arg \max _{k}$ $\alpha_{i k}$, or the dosage $d_{i} \hat{=} \alpha_{i 1}+2 \alpha_{i 2}$, for the genotype in the disease model.

\section{Score Test Using the Posterior Probability Vector}

Intuitively, we anticipate a loss of information when summarizing the entire posterior probability vector with a one-dimensional summary statistic. However, we will now show for the commonly used logistic model that a score test using the dosage is equivalent to a score test from a model that incorporates the entire posterior probability vector.

The observed data likelihood of $\tilde{\pi}_{i}$ for a random sample of $n$ individuals is given by

$$
L\left(\tilde{\pi}_{i}\right)=\prod_{i=1}^{n} \tilde{\pi}_{i}^{y_{i}}\left(1-\tilde{\pi}_{i}\right)^{1-y_{i}},
$$

where

$$
\tilde{\pi}_{i}=\sum_{k=0}^{2} \frac{\exp \left(\beta_{0}+\beta_{1} k+\boldsymbol{\gamma}^{\prime} \boldsymbol{z}_{\boldsymbol{i}}\right)}{1+\exp \left(\beta_{0}+\beta_{1} k+\boldsymbol{\gamma}^{\prime} \boldsymbol{z}_{\boldsymbol{i}}\right)} \alpha_{i k}
$$

and $z_{i}$ is a vector of covariates. The mixture results because we have marginalized over $x_{i}$. The score component, denoted $U\left(\beta_{1}\right)$, which is the gradient of the log-likelihood for $\beta_{1}$, is

$$
\begin{aligned}
U\left(\beta_{1}\right) & =\frac{\partial l}{\partial \beta_{1}}=\sum_{i=1}^{n} \frac{\partial l}{\partial \tilde{\pi}_{i}} \frac{\partial \tilde{\pi}_{i}}{\partial \beta_{1}} \\
& =\sum_{i=1}^{n}\left(\frac{y_{i}}{\tilde{\pi}_{i}}-\frac{1-y_{i}}{1-\tilde{\pi}_{i}}\right)\left(\sum_{k=0}^{2} \frac{\exp \left(\beta_{0}+\beta_{1} k+\boldsymbol{\gamma}^{\prime} \boldsymbol{z}_{\boldsymbol{i}}\right)}{1+\exp \left(\beta_{0}+\beta_{1} k+\boldsymbol{\gamma}^{\prime} \boldsymbol{z}_{\boldsymbol{i}}\right)} k \alpha_{i k}\right)
\end{aligned}
$$

where $l(\cdot)$ is the log-likelihood. Thus, under the null hypothesis of no association, $\beta_{1}=0$ and

$$
\tilde{\pi}_{i}=\pi_{0} \text { for } \tilde{\pi}_{i 0}=\frac{\exp \left(\beta_{0}+\boldsymbol{\gamma}^{\prime} \boldsymbol{z}_{\boldsymbol{i}}\right)}{1+\exp \left(\beta_{0}+\boldsymbol{\gamma}^{\prime} \boldsymbol{z}_{\boldsymbol{i}}\right)} .
$$

The score component is:

$$
\begin{aligned}
& U\left(\beta_{1}=0\right) \\
& =\sum_{i=1}^{n}\left(\frac{y_{i}}{\pi_{0}}-\frac{1-y_{i}}{1-\pi_{0}}\right)\left(\alpha_{i 1} \pi_{0}\left(1-\pi_{0}\right)+2 \alpha_{i 2} \pi_{0}\left(1-\pi_{0}\right)\right) \\
& =\sum_{i=1}^{n}\left(y_{i}\left(1-\pi_{0}\right)-\left(1-y_{i}\right) \pi_{0}\right)\left(\alpha_{i 1}+2 \alpha_{i 2}\right)=\sum_{i=1}^{n}\left(y_{i}-\pi_{0}\right) d_{i} .
\end{aligned}
$$

Next, we consider the score component for the model which substitutes the dosage for the unknown genotype. The model utilizing the dosage can be written as

$$
\pi_{i}=\frac{\exp \left(\beta_{0}+\beta_{1} d_{i}+\boldsymbol{\gamma}^{\prime} \boldsymbol{z}_{\boldsymbol{i}}\right)}{1+\exp \left(\beta_{0}+\beta_{1} d_{i}+\boldsymbol{\gamma}^{\prime} \boldsymbol{z}_{\boldsymbol{i}}\right)} .
$$


In this case, the score component is

$$
\begin{aligned}
U\left(\beta_{1}\right) & =\frac{\partial l}{\partial \beta_{1}}=\sum_{i=1}^{n} \frac{\partial l}{\partial \tilde{\pi}_{i}} \frac{\partial \tilde{\pi}_{i}}{\partial \beta_{1}} \\
& =\sum_{i=1}^{n}\left(\frac{y_{i}}{\tilde{\pi}_{i}}-\frac{1-y_{i}}{1-\tilde{\pi}_{i}}\right)\left(\frac{\exp \left(\beta_{0}+\beta_{1} d_{i}\right)}{\left(1+\exp \left(\beta_{0}+\beta_{1} d_{i}\right)\right)^{2}} d_{i}\right) .
\end{aligned}
$$

And thus,

$$
\begin{aligned}
U\left(\beta_{1}=0\right) & =\sum_{i=1}^{n}\left(\frac{y_{i}}{\pi_{i 0}}-\frac{1-y_{i}}{1-\pi_{i 0}}\right)\left(\pi_{i 0}\left(1-\pi_{i 0}\right) d_{i}\right) \\
& =\sum_{i=1}^{n}\left(y_{i}-\pi_{i 0}\right) d_{i},
\end{aligned}
$$

where again

$$
\tilde{\pi}_{i 0}=\frac{\exp \left(\beta_{0}+\boldsymbol{\gamma}^{\prime} \boldsymbol{z}_{\boldsymbol{i}}\right)}{1+\exp \left(\beta_{0}+\boldsymbol{\gamma}^{\prime} \boldsymbol{z}_{\boldsymbol{i}}\right)} .
$$

We note that a score test is the score component $U$ divided by the negative derivative of the score component $I$, which is known as the observed information. Thus, because $U$ is equivalent and the null models under either formulation admit the same distribution for $U$, it follows that the score tests for association are equivalent. When covariates are present, $U$ and $I$ depend on $\gamma$. The score statistic is computed by substituting $\hat{\gamma}_{0}$, the MLE estimated under the null model, in place of $\gamma$.

Optimal Choice of a One-Dimensional Summary Statistic

While we have demonstrated that the score test using the dosage is equivalent to a score test using the entire vector of posterior probabilities, we have not considered the power of such an approach. The score test is known to perform well asymptotically, but we have no reason to assume that the dosage will perform well in finite samples. In the following subsections, we explore intuitive choices for $\boldsymbol{g}$, a general one-dimensional summary of the posterior probabilities, and then derive its optimal value.

Explicit Expression for the Non-Centrality Parameter

In Appendix A, we assume a logistic model and provide the score statistic for any one-dimensional summary $\boldsymbol{g}$ of $\boldsymbol{x}$, the true genotype. We derive the statistic under the logistic model because this is the most popular model choice in applied work. All subsequent power calculations are performed with respect to this class of test statistics. Our goal is to evaluate the power across a range of true models, which may or may not be logistic, seeing how the optimal summary depends on various assumptions about the true model. An expression is given for the non-centrality parameter when the true model has a general form, $\pi_{i}=f\left(x_{i}, \beta\right)$, and an analytically useful version results when we assume the true model is linear. Since a larger non-centrality parameter is a necessary and sufficient condition for a more powerful test, in the proceeding subsections, we will use the non-centrality parameter to compare the efficiency of various one-dimensional summaries $g$.

\section{Dosage Beats Mode}

In Appendix B, we prove that the dosage is more highly correlated with the true genotype than the mode. This result makes no assumptions about the form of the true disease model (e.g. linear or logistic) and holds for all finite sample sizes. We note, however, that a higher correlation between the imputed genotype and the true genotype does not automatically imply a more powerful test. To draw conclusions about higher power from higher correlation alone, one must also assume that the true disease model is linear: $\pi_{i}=\beta_{0}+\beta_{1} x_{i}$. We consider non-linear models more explicitly later (see subsection Non-Linear Disease Models and Covariates).

\section{Optimal Summary of Posterior Distribution}

Although the superiority of the dosage to the mode is an important result, we have not yet demonstrated that the dosage is an optimal one-dimensional summary statistic. In Appendix C, we show that the score test which results from using the dosage is essentially identical to a score test using the optimal one-dimensional statistic. There, we start by noting that the optimal one-dimensional statistic yields the test with the largest non-centrality parameter. Finding the optimal statistic is therefore equivalent to finding the statistic which maximizes the non-centrality parameter defined in Appendix A. Results from perturbation theory show that the dosage is nearly identical to the optimal statistic in all realistic situations. When the true disease model is linear, it follows that the score test using the dosage is essentially optimal.

Taylor series expansions show that an additive logistic disease model is well approximated by a linear model, when disease prevalence is low for all genotype groups or the SNP effect size is small. Additionally, we expect the projection of the additive logistic model onto the space of all linear models to result in a model respecting the range of $\pi_{i}$, namely some subset of the unit interval, since $x_{i}$ is bounded and only takes three values. For cases in which this linear approximation is inadequate, our results in the subsection Score Test Using the Posterior Probability Vector still apply. These results serve as insurance that the dosage will perform strongly in the case of an additive logistic model, and that it will be asymptotically optimal since the score test is asymptotically most powerful. However, there is no longer any claim of optimality in finite samples when the additive logistic model is not well approximated by a linear model.

\section{Non-Linear Disease Models and Covariates}

The previous sections assumed either a linear or approximately linear disease model. While the assumption of approximate linearity is common in practice with the use of a logistic model, we now consider non-linear modes of inheritance. We first analyze the situation without covariates. In Appendix D, we derive the optimal score test for this case. As in the case of a linear model, the optimal statistic for a non-linear model is well approximated by a simple one-dimensional statistic. Specifically, an approximation of the optimal statistic is given by a linear combination of the dosage and a generalization of the dosage which we call the second-order dosage. This linear combination is given by $d_{i}^{(1)}+\xi d_{i}^{(2)}$, where $d_{i}^{(j)}=\alpha_{i 1}+2^{j} \alpha_{i 2}$ represents the $j$-th order dosage (which governs the $j$-th order effect) for $j=1,2$, and $\xi$ represents the ratio of the second to first order effects, a measure of non-linearity.

To build some intuition about $\xi$, let us examine 2 common nonlinear models. For a dominant disease model, use $\alpha_{1 i}+\alpha_{2 i}$ instead of the dosage, and for a recessive disease model, use $\alpha_{2 i}$ instead of the usual dosage. For more complex models, we can formulate the intuition behind $\xi$ as follows. The posterior distribution of the genotype can be indexed by 2 parameters: the mean and the variance. For linear and approximately linear models, one can simply pretend that $x_{i}=d_{i}$ with little cost. For non-linear models, the cost is 


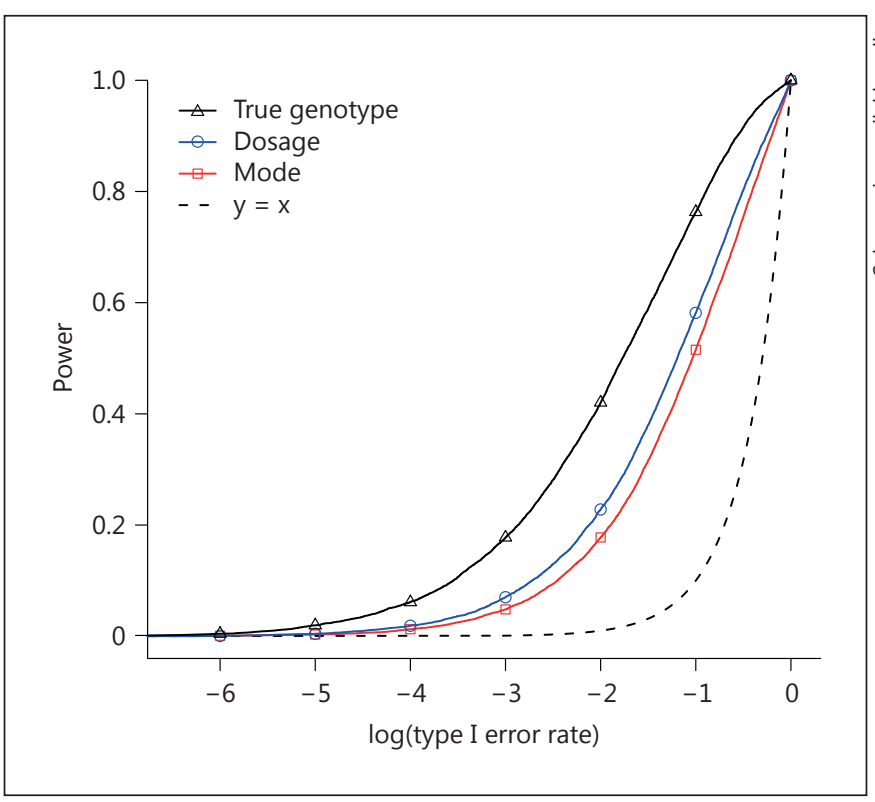

Fig. 1. ROC curve evaluating the asymptotic significance of different approaches to summarizing posterior probability vectors. The asymptotic power of the dosage approach dominates the power of the test when using the mode, for all type I error rates. The graph illustrates the power of a test of case-control association for a SNP with $\mathrm{MAF}=0.10$, odds ratio $=1.28$, and 1,000 cases and 1,000 controls. In this case the imputation $r^{2}$ was 0.60 . The relatively low imputation $r^{2}$ explains why the dosage and mode are not performing better relative to the power of the test when using the true genotype.

non-trivial. The variance in the posterior distribution should then inform us on an individual basis of the cost of the assumption $x_{i}=$ $d_{i}$, and allow us to adjust the weight of evidence accordingly.

In Appendix D, we show that the only change to our analysis through the inclusion of covariates is to allow the non-linearity of the SNP effect to depend on the values of the covariates. Implementation-wise, the optimal linear combination is now $d_{i}^{(\mathrm{f})}+$ $\xi\left(z_{i}\right) d_{i}^{(2)}$, where the measure of effect non-linearity $\xi$ depends on $z_{i}$. Such complications can be avoided if the SNP and covariate effects are additively separable, $\pi_{i}=g\left(x_{i}\right)+h\left(\boldsymbol{z}_{i}\right)$, in which case a common $\xi$ suffices to summarize non-linearity of the SNP effect.

\section{Continuous Traits}

In Appendix E, we derive the optimal score test for normally distributed continuous traits which are linear functions of the genotype and a set of covariates. Thus, we have analytically shown that the dosage is the optimal statistic for the additive continuous traits model considered in Zheng et al. [10].

\section{Simulation}

To verify the theoretical results empirically, we calculated power using simulated data. We considered 3 different characteristics of SNPs: (1) the $r^{2}$ coefficient between the dosages and the genotype (note: this $r^{2}$ coefficient is the value that $\mathrm{MaCH}$ approximates with its $r^{2}$ imputation quality measure [6]), (2) the minor allele frequency (MAF), which unless otherwise stated was set at 0.1 , and (3) the odds ratio under an additive disease model. We consider values of $r^{2}$ ranging from 0.1 to 1 , MAF ranging from 0.05 to 0.5 and odds ratios ranging from 1.0 to 2.0. For each simulation setting, 10,000 SNPs were simulated with 1,000 cases and 1,000 controls. Disease prevalence was fixed at 50\% among individuals with no risk alleles. Unless otherwise stated, power was calculated at the $5 \%$ significance level using the asymptotic distributions of the score tests.

For each SNP and each individual $i$, we compute posterior probabilities $\alpha_{i}$ by sampling from a Dirichlet distribution, where

$$
\alpha_{i}=\left(\alpha_{i 0}, \alpha_{i 1}, \alpha_{i 2}\right) \sim \text { Dirichlet }\left(\frac{(1-q)^{2}}{c}, \frac{2 q(1-q)}{c}, \frac{q^{2}}{c}\right) \text {. }
$$

We let $q$ indicate the MAF of the SNP and $c$ be a non-negative constant chosen to obtain the desired $r^{2}$. Varying $c$ does not appear to greatly modify the standard deviation of the $r^{2}$ coefficient, which ranges between 0.02 and 0.03 across all simulations. An individual's genotype $x_{i}$ was determined by sampling from a multinomial distribution with probabilities indicated by the vector of posterior probabilities $\alpha_{i}$.

\section{Results}

We conducted simulation analyses to confirm the theoretical findings described earlier. In the following section, we briefly describe the results of these simulation analyses. Figure 1 empirically demonstrates that the score test for the dosage is uniformly more powerful than the test for mode. In this setting we note that the power for the true genotype test is much higher than the power for both the dosage and mode tests. However, this is not surprising given the relatively low imputation quality $\left(r^{2}=\right.$ $0.6)$ used in this graph.

As expected, as imputation quality increases, power increases (fig. 2). Furthermore, the power of the dosage and mode tests approaches the power of the linear trend test using the true genotype as $r^{2}$ increases. We note that, for $r^{2}=1$, all 3 tests are identical and thus obtain the same power. On the other hand, for low $r^{2}$ values, the dosage and mode contain little information about the true genotype, and so low power is obtained. Nonetheless, it is interesting to see that the dosage outperforms the mode even in this setting.

Figure 3 shows that the power of all methods increases as the log odds ratio increases. When the odds ratio is 1 , the power is equivalent to the type I error rate. Importantly, all methods control type I error with empirical type I error rates equal to the nominal rate of 0.05 (detailed results not shown). For larger odds ratios, we find 
the expected result that true genotypes are more powerful than dosages, which are in turn more powerful than the mode. As odds ratios grow sufficiently large, all methods have power approaching 1 , though the power rankings of the 3 methods remains.

Lastly, figure 4 shows that power decreases as the MAF decreases. While all tests of association have low power at a MAF of 0.05 , the relative power ordering still holds for the methods, with the true genotype yielding the highest power at 0.40 , the dosage yielding the second highest power at 0.26 , and the mode yielding the lowest power at 0.22 .

We also compared the dosage to the optimal summary statistic given as a dominant eigenvector in Appendix C. Simulations showed that the power and type I error were virtually identical using the two statistics (detailed results not shown).

\section{Discussion}

Previous work has shown that the computational overhead may not be worth the modest power gain from using the entire vector of posterior probabilities instead of the weighted mean posterior probability (dosage). In our analysis, we provided analytic proof that the dosage is essentially equivalent to the optimal choice of a single summary statistic in all practical situations across a range of genetic disease models, far exceeding the power obtained from using the modal posterior probability. These results were confirmed via simulation.

There are a number of important implications of these conclusions. First, while theoretical results and simulations considered the score test, due to the asymptotic equivalence, the optimality of the dosage extends to the related likelihood ratio and Wald tests. Furthermore, as considered in Appendix D, extensions to models including covariates show similar results, unless the effects of covariates are very large.

Superficially, our results may seem to depend on (1) the assumption that the true model is linear or approximately linear, and (2) asymptotic approximations via Taylor expansions. However, 2 facts show that our results should have broad applicability across a range of models and for finite samples. First, the dosage is the one-dimensional summary most highly correlated with the true genotype. This holds for all sample sizes and regardless of the true disease model. Second, naively assuming

$$
\pi_{i}=\frac{\exp \left(\beta_{0}+\beta_{1} d_{i}\right)}{1+\exp \left(\beta_{0}+\beta_{1} d_{i}\right)}
$$

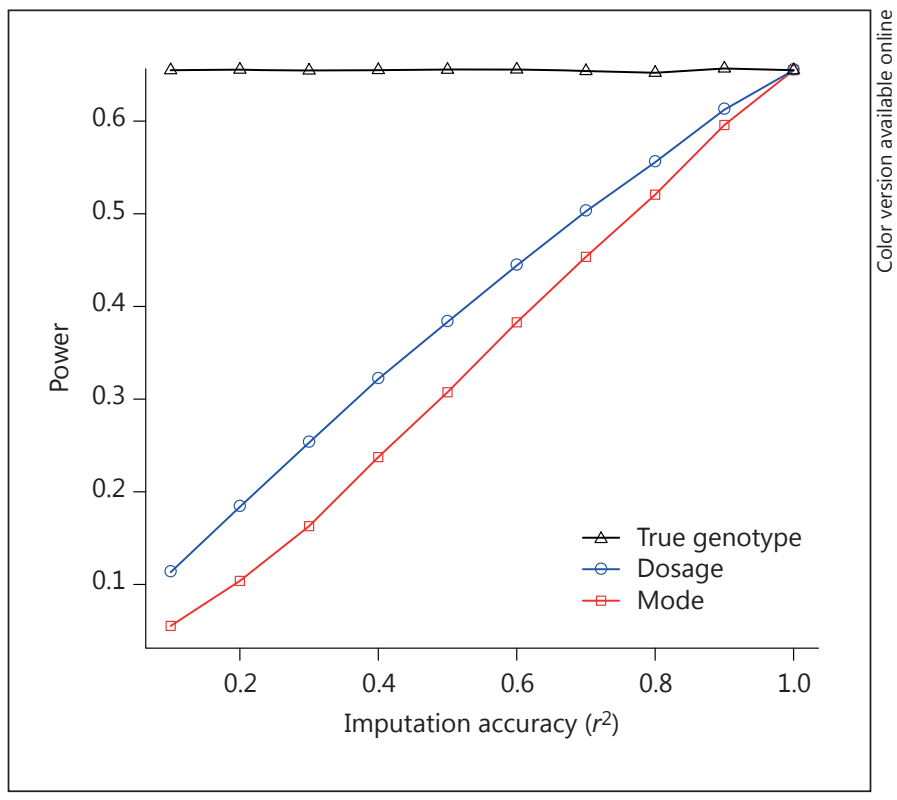

Fig. 2. Evaluating the power of the dosage and mode across different levels of imputation accuracy. Regardless of imputation accuracy, the dosage provides a more powerful choice of summary statistic than the mode. As expected, power increases as imputation accuracy increases. The graph illustrates the power of a test of casecontrol association for a SNP with $\mathrm{MAF}=0.10$, odds ratio $=1.28$, and 1,000 cases and 1,000 controls.

in place of the actual mixture model

$$
\pi_{i}=\sum_{k=0}^{2} \frac{\exp \left(\beta_{0}+\beta_{1} k\right)}{1+\exp \left(\beta_{0}+\beta_{1} k\right)} \alpha_{i k}
$$

does not change the resulting score statistic. Since it is well-known that an additive logistic disease model is robust to misspecification [11], this suggests that the score statistic from the naive model $\pi_{i}=f\left(d_{i}\right)$ well approximates the score statistic from the true mixture model

$$
\pi_{i}=\sum_{i=0}^{2} f(k) \text {. }
$$

Additionally, as shown in Appendix D, in many realistic non-linear models, the dosage remains a nearly optimal choice of one-dimensional statistic because the degree of non-linearity is negligible. Even when the nonlinearity of the SNP effect is appreciable, Appendix D shows that, to the extent that the non-linearity can be explicitly described, simple adjustments to the optimal onedimensional summary can be made to preserve efficiency. Trivially, for a dominant disease model, use $\alpha_{1 i}+\alpha_{2 i}$ instead of the dosage, and for a recessive disease model, use $\alpha_{2 i}$ instead of the usual dosage. A method for choosing 
Fig. 3. Evaluating the power of the dosage and mode across different values of the odds ratio. For both small and large odds ratios, the dosage provides a more powerful alternative to the mode. The graph illustrates the power of a test of case-control association for a SNP with MAF $=0.10$, imputation $r^{2}=0.6$, and 1,000 cases and 1,000 controls.

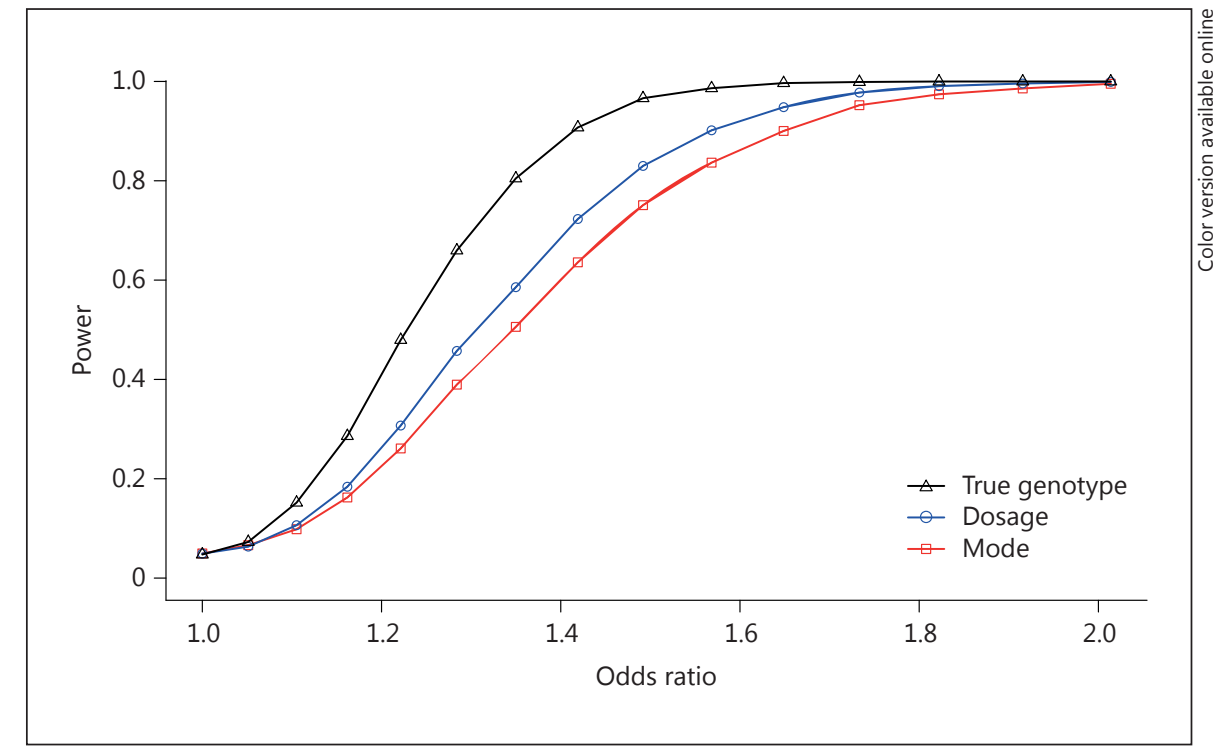

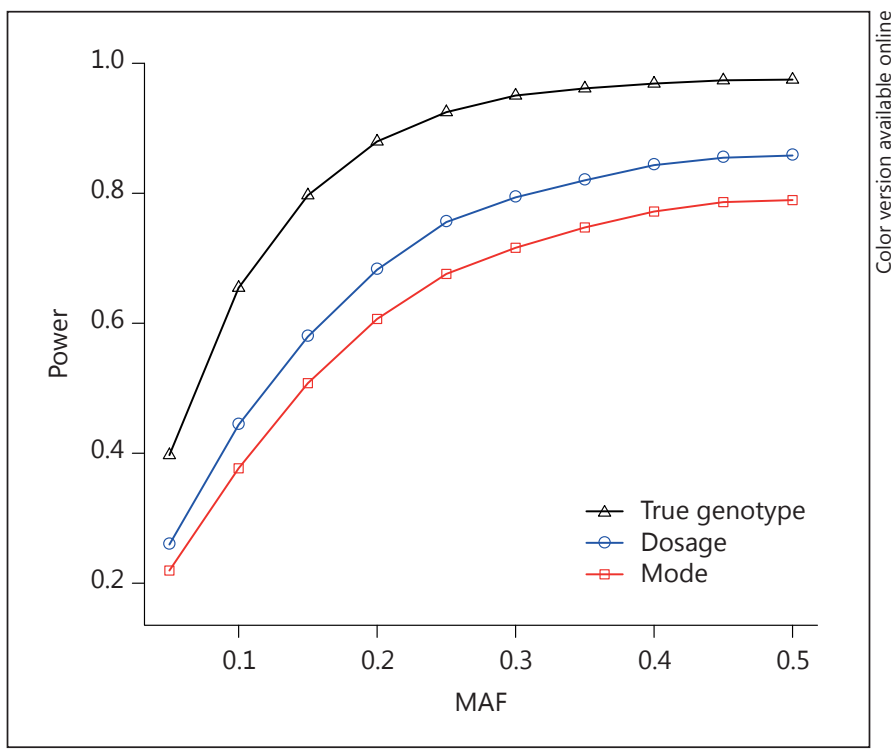

Fig. 4. Evaluating the power of the dosage and mode across different MAFs. Across all MAFs, the dosage provides a more powerful alternative to the mode. The graph illustrates the power of a test of case-control association for a SNP with odds ratio of 1.25 , imputation $r^{2}=0.6$, and 1,000 cases and 1,000 controls.

the optimal summary statistic for more complex models can be found in Appendix D. In additional simulation analyses, not shown here, the dosage showed robustness across a wide variety of non-linear models, with robustness related to the extent of non-linearity.

Optimal Methods for Using Posterior Probabilities
For continuous responses, Zheng et al. [10] implemented a mixture model utilizing the entire vector of posterior probabilities. In analyses not reported here, we proceeded similarly with an E-M algorithm and found, like Zheng et al., that dosage performed similarly with far less computational expense. In Appendix E, we outline the proof of optimality of the dosage under a normal linear model for a quantitative response. While we did not provide simulations here, an extensive consideration of this model and deviations from it can be found in Zheng et al. [10]. These extensive simulations also consider cases with sample sizes as low as 50 (figure 3 in Zheng et al. [10]), suggesting robustness to the asymptotic assumptions underlying portions of the results shown here.

While we do not consider the new class of rare variant tests explicitly, our results may be extendable to 2 classes of rare variant tests, with a word of caution. For rare variant tests which collapse rare variants into a single 'super variant', the dosage is given by the probability that any one of the included variant sites contains the rare variant, i.e. by $1-\Pi_{j} \alpha_{0 i j}$, where $\alpha_{0 i j}$ represents the posterior probability for individual $i$ not to have a rare variant at site $j$. For rare variant tests which regress the total number of rare variants present across a set of variant sites, the modified dosage is given by $\Sigma_{j} d_{i j}$. The former is really a special case of the latter, based on the approximation $\sum_{j} x_{i j} \approx$ $1\left\{\sum_{j} x_{i j}>1\right\}$. Suppose that the variants enter into the disease model additively:

$$
\sum_{j} \beta_{j} x_{i j}=\beta \sum_{j} \frac{\beta_{j}}{\beta} x_{i j} .
$$


Then our results apply by thinking about the imputation of

$$
\sum_{j} \frac{\beta_{j}}{\beta} x_{i j}
$$

which now depends not only on the posterior probabilities for each $x_{i j}$, but also on the nuisance parameters

$$
\frac{\beta_{j}}{\beta} \text {. }
$$

How to effectively estimate the nuisance parameters remains an area of active research. However, given the nuisance parameters, our results suggest that a nearly optimal summary would be

$$
\sum_{j} \frac{\beta_{j}}{\beta} d_{i j} \text {. }
$$

In particular, one may justify the use of $\sum_{j} d_{i j}$ by assuming homogeneity of effects across variants. Caution needs to be taken for small sets of variant sets or sample sizes, however, because in these cases the perturbation term by which the dosage differs from the optimal summary may be non-trivial. Simulation studies and further analyses of these rare variant strategies, along with considerations of the recently proposed class of variance-component tests, are needed.

Few assumptions are required on the posterior probabilities in order for the results described here to be valid. In particular, posterior probabilities, while commonly obtained from imputation, can also be obtained from both SNP microarray and next-generation sequencing technologies. The analytic calculations shown here directly extend to these platforms. The main necessary assumption about the posterior probabilities is that they are correctly calibrated - namely, that the vector of posterior probabilities $\alpha_{i}$ can be interpreted as suggesting that the true minor allele count for individual $i$, denoted $x_{i}$, can be modeled as being a single random draw from a multinomial distribution with probabilities indicated by $\alpha_{i}$. While this interpretation is almost uniformly made in practice, any systematic technological bias could impact this interpretation, making the analytic conclusions provided above no longer hold.

The dosage is commonly used as a shortcut to the use of a wide class of statistical methods, which assume knowledge of the true genotypes. We provide analytic justification of its use across a wide variety of genetic models.

\section{Appendix A}

Much of the derivation of the non-centrality parameter follows $\mathrm{Wu}$ et al. [12]. The logistic model utilizing a general one-dimensional summary of the posterior genotype distribution $g_{i}$ can be written as $y_{i} \mid g_{i} \sim \operatorname{Bern}\left(\pi_{i}\left(g_{i}\right)\right)$, where $\operatorname{logit}\left(\pi_{i}\right)=\beta_{0}+\beta_{1} g_{i}$. Following arguments made in the main text (see Methods - Score Test Using the Posterior Probability Vector), we can denote the squared score component as $\left(\boldsymbol{y}-\hat{\boldsymbol{\pi}}_{\mathbf{0}}\right)^{\prime} \mathbf{g g}^{\prime}\left(\boldsymbol{y}-\hat{\boldsymbol{\pi}}_{\mathbf{0}}\right)$, where $\boldsymbol{y}=\left(y_{1}, \ldots, y_{\mathrm{n}}\right)^{\prime}, \boldsymbol{g}=\left(g_{1}, \ldots\right.$, $\left.g_{n}\right)^{\prime}$, and $\hat{\pi}_{0}$ is the vector of disease probabilities estimated under the null hypothesis. We note that when the SNP is typed, $g_{i}=x_{i}$ and the above corresponds to the Armitage linear trend test.

Define $\mu_{\beta} \bumpeq\left(\pi_{1}-\pi_{0}, \ldots, \pi_{n}-\pi_{0}\right)^{\prime}$ as the vector of differences between the true disease probability and the null disease probability. Under the null hypothesis, we have $\boldsymbol{\mu}_{\beta}=\mathbf{0}_{n}$. Let $\boldsymbol{V} \triangleq \operatorname{Cov}(\boldsymbol{y})=$ $\operatorname{diag}\left(\pi_{i}\left(1-\pi_{i}\right)\right)$. The squared score component can be rewritten as follows:

$$
\begin{aligned}
& \left(y-\hat{\pi}_{0}\right)^{\prime} g g^{\prime}\left(y-\hat{\pi}_{0}\right) \\
& =\left(y-\hat{\pi}_{0}-\mu_{\beta}+\mu_{\beta}\right)^{\prime} V^{-1 / 2} V^{1 / 2} g g^{\prime} V^{1 / 2} V^{-1 / 2}\left(y-\hat{\pi}_{0}-\mu_{\beta}+\mu_{\beta}\right) \\
& =\left(z+\widetilde{\mu}_{\beta}\right)^{\prime} K\left(z+\widetilde{\mu}_{\beta}\right),
\end{aligned}
$$

where $z \triangleq \boldsymbol{V}^{-1 / 2}\left(\boldsymbol{y}-\hat{\boldsymbol{\pi}}_{\mathbf{0}}-\boldsymbol{\mu}_{\beta}\right), \tilde{\boldsymbol{\mu}}_{\boldsymbol{\beta}} \triangleq \boldsymbol{V}^{-1 / 2} \boldsymbol{\mu}_{\beta}$, and $\boldsymbol{K} \triangleq \boldsymbol{V}^{1 / 2} \boldsymbol{g} \boldsymbol{g}^{\prime} \boldsymbol{V}^{1 / 2}$. Note that $z_{i}$ has mean 0 and variance 1 . A spectral decomposition on $\boldsymbol{K}$ gives $\boldsymbol{K}=\boldsymbol{U} \boldsymbol{\Lambda} \boldsymbol{U}^{\prime}$, where $\boldsymbol{U}=\left(\boldsymbol{u}_{1}, \ldots, \boldsymbol{u}_{\boldsymbol{n}}\right)$ and $\boldsymbol{\Lambda}=\operatorname{diag}\left(\lambda_{1}, \ldots, \lambda_{n}\right) \cdot \boldsymbol{u}_{i}$ are the orthonormal eigenvectors of $\boldsymbol{K}$ corresponding to the eigenvalues $\lambda_{i}$. Because $g$ is $n \times 1$, only one of the eigenvalues is nonzero, and we take this to be $\lambda_{1}$. Asymptotically, $\boldsymbol{u}_{1}^{\prime}\left(z+\tilde{\boldsymbol{\mu}}_{\beta}\right) \sim N\left(\boldsymbol{u}_{1}^{\prime} \tilde{\boldsymbol{\mu}}_{\beta}\right.$, 1). Thus,

$$
\left(y-\hat{\pi}_{0}\right)^{\prime} g g^{\prime}\left(y-\hat{\pi}_{0}\right)=\left(z+\tilde{\mu}_{\beta}\right)^{\prime} U \Lambda U^{\prime}\left(z+\tilde{\mu}_{\beta}\right) \sim \lambda_{1} \chi_{1}^{2}(\delta),
$$

where $\delta$ is the non-centrality parameter, and $\delta \hat{=} \tilde{\boldsymbol{\mu}}_{\beta} \boldsymbol{u}_{1} \boldsymbol{u}_{\mathbf{1}}^{\prime} \tilde{\boldsymbol{\mu}}_{\beta}=$ $\left(\boldsymbol{u}_{\mathbf{1}}^{\prime} \boldsymbol{\mu}_{\beta}\right)^{2}$. Note that $\boldsymbol{u}_{\mathbf{1}} \cdot \tilde{\boldsymbol{\mu}}_{\beta}=\cos \theta\left\|\boldsymbol{u}_{\mathbf{1}}\right\|\left\|\tilde{\boldsymbol{\mu}}_{\beta}\right\|=\cos \theta\left\|\tilde{\boldsymbol{\mu}}_{\beta}\right\|$, since $\boldsymbol{u}_{1}$ is orthonormal and $\cos \theta\left\|\tilde{\boldsymbol{\mu}}_{\beta}\right\|$ is simply the length of the projection of $\tilde{\boldsymbol{\mu}}_{\beta}$ in the direction of $\boldsymbol{u}_{\mathbf{1}}$. In this case

$$
u_{1}=\frac{V^{1 / 2} \boldsymbol{g}}{\left\|V^{1 / 2} \boldsymbol{g}\right\|} \text {. }
$$

Thus the quantity which determines power is $\cos \theta$, where $\theta$ is the angle between $\boldsymbol{V}^{1 / 2} \boldsymbol{g}$ and $\tilde{\boldsymbol{\mu}}_{\beta}$, i.e. how well the summary of the posterior genotype distribution $\boldsymbol{g}$ is aligned with the ways in which the truth deviates from the null hypothesis, $\widetilde{\boldsymbol{\mu}}_{\beta}=\boldsymbol{\pi}-\hat{\boldsymbol{\pi}}_{0}$.

Consider the special case of a disease model which is truly linear, $\pi_{i}=\pi_{0}+\beta_{1} x_{i}$. Under this model, $\tilde{\boldsymbol{\mu}}_{\beta}=\beta_{1} \boldsymbol{x}$. The non-centrality parameter is $\left(\boldsymbol{u}_{\mathbf{1}}^{\prime} \tilde{\boldsymbol{\mu}}_{\beta}\right)^{2}=\left(\beta_{1} \boldsymbol{g}^{\prime} \boldsymbol{x}\right)^{2}=\left(\beta_{1} \cos \theta_{\boldsymbol{g}, \boldsymbol{x}}\|\boldsymbol{g}\|\|x\|\right)^{2}$. Note that the key quantity is $\cos \theta_{g, x}$ or how well aligned our summary $g$ is with the true genotype vector $\boldsymbol{x}$.

\section{Appendix B}

We now consider the special cases of the score test from Appendix $\mathrm{A}$, where the one-dimensional summary statistic $g_{i}$ is equal to $d_{i}$ (dosage) or $m_{i}$ (mode). $\cos \theta_{g, x}$ is then the observed correlation between $g_{i}$ and the true genotype $x_{i}$, and we show that the dosage is always more highly correlated with the true genotype than the mode is. Here, we assume that the posterior probability vectors $\alpha_{i}=\left(\alpha_{0 i}, \alpha_{1 i}, \alpha_{2 i}\right)$ are drawn i.i.d. from some arbitrary distribution on the 2 -simplex. We note that this implies that $d_{i}$ and $m_{i}$ are now both random variables, whereas above when $\alpha_{i}$ was treated as fixed, 
the dosage and mode were also fixed. In other sections of this paper, we condition on $\alpha_{i}$ and thus are able to treat them as constant.

Using the law of total covariance

$$
\begin{aligned}
& \operatorname{Cov}\left(x_{i}, d_{i}\right)=E\left(\operatorname{Cov}\left(x_{i}, d_{i} \mid \alpha_{i}\right)\right)+\operatorname{Cov}\left(E\left(x_{i} \mid \alpha_{i}\right), E\left(d_{i} \mid \alpha_{i}\right)\right) \\
& =\operatorname{Var}\left(d_{i}\right)
\end{aligned}
$$

because given $\alpha_{i}, d_{i}$ is a constant and thus $\operatorname{Cov}\left(x_{i}, d_{i} \mid \alpha_{i}\right)=0$ and $d_{i}=E\left(x_{i} \mid \alpha_{i}\right)$. A second application of the law of total covariance gives:

$$
\begin{aligned}
& \operatorname{Cov}\left(x_{i}, m_{i}\right)=E\left(\operatorname{Cov}\left(x_{i}, m_{i} \mid \alpha_{i}\right)\right)+\operatorname{Cov}\left(E\left(x_{i} \mid \alpha_{i}\right), E\left(m_{i} \mid \alpha_{i}\right)\right) \\
& =\operatorname{Cov}\left(d_{i}, m_{i}\right)
\end{aligned}
$$

Let $r_{\text {mean }}$ represent $\operatorname{Cor}\left(x_{i}, d_{i}\right)$ and $r_{\text {mode }}$ represent $\operatorname{Cor}\left(x_{i}, m_{i}\right)$. Thus, by substitution, we have:

$$
\begin{aligned}
r_{\text {mean }}^{2}-r_{\text {mode }}^{2} & =\frac{\operatorname{Cov}\left(x_{i}, d_{i}\right)}{\sqrt{\operatorname{Var}\left(x_{i}\right) \operatorname{Var}\left(d_{i}\right)}}-\frac{\operatorname{Cov}\left(x_{i}, m_{i}\right)}{\sqrt{\operatorname{Var}\left(x_{i}\right) \operatorname{Var}\left(m_{i}\right)}} \\
& =\frac{\operatorname{Var}\left(d_{i}\right) \sqrt{\operatorname{Var}\left(m_{i}\right)}-\operatorname{Cov}\left(x_{i}, m_{i}\right) \sqrt{\operatorname{Var}\left(d_{i}\right)}}{\sqrt{\operatorname{Var}\left(x_{i}\right) \operatorname{Var}\left(d_{i}\right) \operatorname{Var}\left(m_{i}\right)}} \\
& =\frac{\sqrt{\operatorname{Var}\left(d_{i}\right) \operatorname{Var}\left(m_{i}\right)}-\operatorname{Cov}\left(d_{i}, m_{i}\right)}{\sqrt{\operatorname{Var}\left(x_{i}\right) \operatorname{Var}\left(m_{i}\right)}} \\
& \geq 0
\end{aligned}
$$

since

$$
\operatorname{Cov}\left(d_{i}, m_{i}\right) \leq \sqrt{\operatorname{Var}\left(d_{i}\right) \operatorname{Var}\left(m_{i}\right)}
$$

by the Cauchy-Schwarz inequality.

So far, no asymptotic arguments have been used. They come into play only when linking $r_{\text {mean }}^{2}$ to $\cos ^{2} \theta_{d, x}$ and $r_{\text {mode }}^{2}$ to $\cos ^{2} \theta_{m, x}$, where $r_{\text {mean }}^{2}$ and $r_{\text {mode }}^{2}$ are the population counterparts of the sample quantities $\cos ^{2} \theta_{d, x}$ and $\cos ^{2} \theta_{m, x}$. The justification for using dosage over mode in any particular sample depends on the inequality $\cos ^{2} \theta_{\boldsymbol{d}, \boldsymbol{x}} \geq \cos ^{2} \theta_{\boldsymbol{m}, \boldsymbol{x}}$, rather than the inequality $r_{\text {mean }}^{2} \geq$ $r_{\text {mode. }}^{2}$ As $n \rightarrow \infty, \cos ^{2} \theta_{\boldsymbol{d}, \boldsymbol{x}} \rightarrow r_{\text {mean }}^{2}$ and $\cos ^{2} \theta_{m, x} \rightarrow r_{\text {mode }}^{2}$ [13]. Thus as $n \rightarrow \infty, \cos ^{2} \theta_{d, x} \geq \cos ^{2} \theta_{m, x}$ almost surely. And so, based on the equation for the non-centrality parameter shown in Appendix A, the test using the dosage has a larger non-centrality parameter than the test using the mode, implying that the score test using the dosage has more power than the score test using the mode.

\section{Appendix C}

For the set of linear trend tests using the statistic $\left(\boldsymbol{y}-\hat{\boldsymbol{\pi}}_{\mathbf{0}}\right)^{\prime} \boldsymbol{g} \boldsymbol{g}^{\prime}$ $\left(\boldsymbol{y}-\hat{\boldsymbol{\pi}}_{0}\right)$, we derive the optimal $\boldsymbol{g}$, where we define optimal to be the $g$ which yields the most powerful score test. Thus, to find the optimal $\boldsymbol{g}$, we wish to maximize the non-centrality parameter $\boldsymbol{u}_{1}^{\prime} \tilde{\boldsymbol{\mu}}_{\beta} \widetilde{\boldsymbol{\mu}}_{\beta}^{\prime} \boldsymbol{u}_{1}$ (see Appendix A), subject to the constraint that $\left\|u_{1}\right\|=1$. Under the linear disease model, $\tilde{\boldsymbol{\mu}}_{\beta}=\beta_{1} \boldsymbol{V}^{-1 / 2} \boldsymbol{x}$, where $\beta_{1}$ is a multiplicative constant that is irrelevant to the optimization problem. Thus, we wish to solve the optimization problem given as follows:

$$
\max _{u \in \mathbb{R}^{n}} E\left(\boldsymbol{u}^{\prime}\left(\boldsymbol{V}^{-1 / 2} \boldsymbol{x}\right)\left(\boldsymbol{V}^{-1 / 2} \boldsymbol{x}\right)^{\prime} \boldsymbol{u}\right) \text { subject to } \boldsymbol{u}^{\prime} \boldsymbol{u}=1 .
$$

Because $\boldsymbol{u}=\boldsymbol{V}^{1 / 2} \boldsymbol{g}$ and the optimal $\boldsymbol{g}$ is unique only up to scaling (the significance of the test does not depend on how we scale $\boldsymbol{g}$ ), we can reformulate the problem as:

Optimal Methods for Using Posterior Probabilities $\max _{\boldsymbol{g} \in \mathbb{R}^{n}} E\left(\boldsymbol{g}^{\prime} \mathbf{x} \mathbf{x}^{\prime} \boldsymbol{g}\right)$ subject to $\boldsymbol{g}^{\prime} \boldsymbol{g}=1$.

The first-order conditions are:

$$
\left(E(\mathbf{x}) E(\boldsymbol{x})^{\prime}+\operatorname{Cov}(\boldsymbol{x})-\lambda \boldsymbol{I}\right) \boldsymbol{g}=0
$$

Thus $g$ is the dominant eigenvector of

$$
E(\mathbf{x}) E(\boldsymbol{x})^{\prime}+\operatorname{Cov}(\boldsymbol{x})=\boldsymbol{d} \boldsymbol{d}^{\prime}+\operatorname{Cov}(\boldsymbol{x}),
$$

where $\operatorname{Cov}(\boldsymbol{x})=\operatorname{diag}\left(\alpha_{1 i}+4 \alpha_{2 i}-d_{i}^{2}\right)$.

Let $\boldsymbol{g}^{*}$ be the dominant eigenvector of $\boldsymbol{d} \boldsymbol{d}^{\prime}$, which ignores the covariance term. Below, we justify why ignoring the covariance term has negligible effect in most situations. Note that

$$
g^{*}=\frac{d}{\|d\|}
$$

and since the scaling of $\boldsymbol{g}$ does not matter for testing purposes, this suggests we take $\boldsymbol{g}=\boldsymbol{g}^{*}=\boldsymbol{d}$, if the covariance term can be ignored.

To justify approximating $g$ with $g^{*}$, in essence this is why it is acceptable to ignore the covariance term, we can assume without loss of generality that

$$
\max _{i}\left[\alpha_{1 i}+4 \alpha_{2 i}-d_{i}^{2}\right]=\operatorname{Var}\left(x_{1}\right) \text {. }
$$

Applying a result from perturbation theory, see equation 6 in Lang et al. [13], yields:

$$
\begin{aligned}
& \frac{1}{2} \sin 2 \theta_{g^{*}, g} \leq \frac{\max _{i} \alpha_{1 i}+4 \alpha_{2 i}-d_{i}^{2}}{\sum_{i} d_{i}^{2}} \leq \frac{\operatorname{Var}\left(x_{1}\right)}{E\left(x_{1}\right)^{2}+\sum_{i=2}^{n} E\left(x_{i}\right)^{2}} \\
& =\left(\frac{1}{C V^{2}\left(x_{1}\right)}+\sum_{i=2}^{n} \frac{\operatorname{Var}\left(x_{i}\right)}{\operatorname{Var}\left(x_{1}\right)} \frac{1}{C V^{2}\left(x_{i}\right)}\right)^{-1}=\left(\sum_{i=1}^{n} w_{i} \frac{1}{C V^{2}\left(x_{i}\right)}\right)^{-1}
\end{aligned}
$$

where $C V$ is the coefficient of variation, where $w_{i} \leq 1$.

We can interpret

$$
\frac{1}{C V^{2}\left(x_{i}\right)}
$$

as a measure of the signal-to-noise ratio (of the imputation process) for individual $i$. Thus,

$$
\sum_{i=1}^{n} w_{i} \frac{1}{C V^{2}\left(x_{i}\right)}
$$

is a weighted sum that measures the overall precision of the imputation process, where the weights

$$
\frac{\operatorname{Var}\left(x_{i}\right)}{\operatorname{Var}\left(x_{1}\right)}
$$

serve as a standardization factor. As $n$ goes to infinity, the weighted sum of the precisions goes to infinity as well, i.e. there is an accumulation of genotype information across individuals so that the angle between $\boldsymbol{g}$ and $\boldsymbol{g}^{*}$ goes to 0 . Finally, we combine the bound with the following approximation for small angles

$$
\theta_{g^{*}, g} \approx \sin \theta_{g^{*}, g} \approx \frac{1}{2} \sin \theta_{g^{*}, g} \approx 0
$$

to conclude that $\boldsymbol{g}^{*}$ and $\boldsymbol{g}$ are essentially identical. 
Finally, we note that, since the $\alpha_{i}$ s are observed, one can always calculate

$$
\frac{1}{C V^{2}\left(x_{i}\right)}
$$

for each study to find an upper bound on how much $g$ and $g^{*}$ are expected to differ. However, we note that in our analyses, details are not shown, for practical sample sizes, $\boldsymbol{g}$ and $\boldsymbol{g}^{*}$ will be very close.

\section{Appendix D}

We now consider more general disease models and study how a non-linear effect of genotype count on disease risk impacts the efficacy of one-dimensional summaries of the posterior probability vector. Let $x_{i}$ be the genotype of individual $i$ and $z_{i}$ be a vector of covariates. Let $\pi_{i}\left(x_{i}, z_{i}\right)=f\left(x_{i}, z_{i}\right)$ and $\pi_{0 i}=f_{0}\left(z_{i}\right)=f\left(0, z_{i}\right)$. Note that the inclusion of covariates in the model suggests that $\pi_{0 i}$ may vary between individuals, unlike in the previous Appendices. Because $x_{i}$ has only 3 states, any $f$ is sufficiently described by a quadratic fit through the 3 points, and so without loss of generality, we assume $f$ is quadratic. Then:

$$
\pi_{i}-\pi_{0 i}=f\left(x_{i}, z_{i}\right)-f_{0}\left(z_{i}\right)=f^{\prime}\left(0, z_{i}\right) x_{i}+f^{\prime \prime}\left(0, z_{i}\right) x_{i}^{2} .
$$

There are 2 assumptions we can make to simplify the analysis: (1) $f$ is sufficiently linear in $x_{i}\left(f^{\prime}\left(0, z_{i}\right)=0\right)$ for all $z_{i}$ values and (2) interactions between $x_{i}$ and $z_{i}$ are negligible $\left(f^{\prime}\left(0, z_{i}\right)=f^{\prime}(0)\right.$ is free of $\left.z_{i}\right)$. A special form of (2) occurs, when we assume that the SNP and covariate effects are additively separable: $\pi_{i}=g\left(x_{i}\right)+h\left(z_{i}\right)$. Then $\pi_{i}-\pi_{0 i}=g\left(x_{i}\right)$, and the problem reduces to the case without covariates. If we use both assumptions (1) and (2), then the intuitions derived in Appendices B and C for a disease model where risk is linear in genotype count are expected to hold. The logistic model belongs to this class, thus explaining why dosage performs so well in this important case. To see this in the simple case of no covariates, let

$$
\pi_{i}=\frac{e^{\beta_{0}+\beta_{1} x_{i}}}{1+e^{\beta_{0}+\beta_{1} x_{i}}} \equiv f\left(x_{i}\right)
$$

and Taylor expand this expression about the dosage, $d_{i}$ :

$$
\pi_{i}=f\left(d_{i}\right)+f^{\prime}\left(d_{i}\right)\left(x_{i}-d_{i}\right)+f^{\prime \prime}\left(d_{i}\right)\left(x_{i}-d_{i}\right)^{2} .
$$

One can show that $f^{\prime \prime}\left(d_{i}\right)=\beta_{1}^{2} \pi_{i}\left(1-\pi_{i}\right)\left(1-2 \pi_{i}\right) \leq 0.1 \beta_{1}^{2}$. A linear approximation to the logistic model results if we can justify ignoring the second-order term. The upper bound already gives us some grounds for doing so. In addition, $f^{\prime \prime}\left(d_{i}\right)$ is near 0 and thus negligible if $\pi_{i} \approx 0$ (i.e. low prevalence in a prospective cohort study), if $\pi_{i} \approx 0.5$ (i.e. an approximately equal number of cases and control in a case-control study), or if the SNP effect is small.

We now move towards an analysis applicable to a general disease model by first relaxing assumption (2), i.e. the effect of $x_{i}$ does not depend on the value of $z_{i}$. Then $\widetilde{\boldsymbol{\mu}}_{\beta}=\boldsymbol{V}^{-1 / 2} \boldsymbol{B} \boldsymbol{x}$, where $\boldsymbol{B}=$ $\operatorname{diag}\left(f^{\prime}\left(0, z_{i}\right)\right)$. The optimization problem for the non-centrality parameter becomes $\max _{\boldsymbol{g} \in \mathbb{R}^{n}} E\left(\boldsymbol{g}^{\prime} \boldsymbol{B} \boldsymbol{x} \boldsymbol{x}^{\prime} \boldsymbol{B} \boldsymbol{g}\right)$ subject to $\boldsymbol{g}^{\prime} \boldsymbol{g}=1$. If $\boldsymbol{B}$ is far from being proportional to the identity matrix, then this implies that the amount of 'signal' we can expect from different individuals is different on average. Thus, the scheme which treats all individuals equally is suboptimal.

We now relax assumption (1), i.e. linearity of the SNP effect. To optimize the non-centrality parameter on average, we solve arg $\max _{\boldsymbol{g} \in \mathbb{R}^{n}} E\left(\boldsymbol{g}^{\prime} \boldsymbol{\mu}_{\beta} \boldsymbol{\mu}_{\beta}^{\prime} \boldsymbol{g}\right)$ subject to $\boldsymbol{g}^{\prime} \boldsymbol{g}=1$. Recalling that $\boldsymbol{\mu}_{\beta}$ represents the vector of differences between the true disease probability and the null disease probability, it follows from the Taylor series expansion that the $n \times 1$ vector $\mu_{\beta}$ is given by $\left(\mu_{\beta i}\right)=\left(f^{\prime}\left(0, z_{i}\right) x_{i}+\right.$ $\left.f^{\prime \prime}\left(0, z_{i}\right) x_{i}^{2}\right)$. Following the results of Appendix $\mathrm{C}$, the solution is the dominant eigenvector of $\boldsymbol{V}^{-1 / 2}\left[E\left(\boldsymbol{\mu}_{\beta}\right) E\left(\boldsymbol{\mu}_{\beta}\right)^{\prime}+\operatorname{Cov}\left(\boldsymbol{\mu}_{\beta}\right)\right] \boldsymbol{V}^{-1 / 2}$.

Using the same arguments from perturbation theory, $E\left(\boldsymbol{\mu}_{\beta}\right)$ is an essentially optimal one-dimensional summary. Here, $E\left(\mu_{\beta i}\right)=$ $E\left(f^{\prime}\left(0, z_{i}\right) x_{i}+f^{\prime \prime}\left(0, z_{i}\right) x_{i}^{2}\right)=f^{\prime}\left(0, z_{i}\right) d_{i}^{(1)}+f^{\prime \prime}\left(0, z_{i}\right) d_{i}^{(2)}$, where $d_{i}^{(j)} \hat{=}$ $E\left(x_{i}^{(j)}\right)=\alpha_{i 1}+2^{j} \alpha_{i 2}$ gives what we call the $j$-th order dosage. We note that in most realistic situations, $E\left(\boldsymbol{\mu}_{\beta}\right)^{\prime} E\left(\boldsymbol{\mu}_{\beta}\right)$ will be large enough that the angle between the dosage vector $E\left(\boldsymbol{\mu}_{\beta}\right)$ and the optimal summary $\boldsymbol{g}$ will be essentially 0 .

Note that $d_{i}^{(1)}$ is denoted $d$ in the rest of this paper. The relative importance of the first-order versus the second-order dosage in our optimal summary is determined by the relative magnitudes of $f^{\prime}\left(0, z_{i}\right)$ and $f^{\prime \prime}\left(0, z_{i}\right)$. If the effect on risk of the second allele differs significantly from that of the first, i.e. $f^{\prime \prime}\left(0, z_{i}\right)$ is large in magnitude, then the first-order dosage is an insufficient summary. This result is intuitive.

The extent to which we need information beyond the dosage depends on the extent to which the disease model is non-linear. Note that $f^{\prime}\left(0, z_{i}\right)$ and $f^{\prime \prime}\left(0, z_{i}\right)$ are not known, so implementing the optimal one-dimensional summary is infeasible for highly non-linear risk models, unless one is willing to make an educated guess about the relative degree of the second-order effect. For example, if we believe that the second-order effect is some fraction $\xi$ of the firstorder effect, $f^{\prime \prime}\left(0, z_{i}\right) \propto \xi f^{\prime}\left(0, z_{i}\right)$ for all $z_{i}$, then an optimal summary would be $d_{i}^{(1)}+\xi d_{i}^{(2)}$. Otherwise, $\xi=\xi\left(z_{i}\right)$ may depend on the covariate value for each individual, and the optimal summary for each individual would weigh the first- and second-order dosages differently, $d_{i}^{(1)}+\xi\left(z_{i}\right) d_{i}^{(2)}$. Note that in most cases $\xi$ is unknown, and the degree of freedom lost by trying to estimate $\xi$ may more than outweigh any efficiency gain from obtaining a better summary of $x_{i}$.

\section{Appendix E}

Suppose now that $y_{i}$ is a quantitative trait. Let $Z=\left(z_{1}, \ldots, z_{n}\right)^{\prime}$, where each $z_{i}$ is a column vector of covariates for individual $i$ of length $l_{z}$. Assume the normal linear model given by: $y_{i}=\mu_{i}+\varepsilon_{i}=$ $\beta_{0}+\beta_{1} x_{i}+\gamma^{\prime} z_{i}+\varepsilon_{i}$, where $\gamma$ is a constant length $l_{z}$ a column vector and $\varepsilon_{i} \sim N\left(0, \sigma^{2}\right)$. Further let $X=(\boldsymbol{x}, Z)$, where $\boldsymbol{x}$ is $n \times 1$. The Fstatistic testing the null hypothesis, $\beta_{1}=0$, is:

$$
\frac{y^{\prime}\left(P_{1}-P_{0}\right) y}{y^{\prime}\left(I-P_{1}\right) y /\left(n-l_{Z}\right)} .
$$

Where $P_{1}=X\left(X^{\prime} X\right)^{-1} X^{\prime}$ and $P_{0}=Z\left(Z^{\prime} Z\right)^{-1} Z^{\prime}$. The non-centrality parameter of the statistic is an increasing function of:

$$
\boldsymbol{\mu}^{\prime}\left(P_{1}-P_{0}\right) \boldsymbol{\mu}=\boldsymbol{x}^{\prime} \beta_{1}\left[P_{1}-P_{0}\right] \beta_{1} \boldsymbol{x} .
$$

$\boldsymbol{x}$ is unknown. Suppose we replace $\boldsymbol{x}$ with $\hat{\boldsymbol{x}}$ (accordingly, $P_{1}$ with $\hat{P}_{1}$ ). The non-centrality parameter for the F-statistic from using $\hat{x}$ is $\boldsymbol{x}^{\prime} \beta_{1}\left[\hat{P}_{1}-P_{0}\right] \beta_{1} \boldsymbol{x}$. To maximize power, we again maximize this non-centrality parameter. That is, we seek to solve the following optimization problem:

$$
\begin{aligned}
& \min _{\hat{x} \in \mathbb{R}^{n}} E\left[\boldsymbol{x}^{\prime} \beta_{1}\left[P_{1}-P_{0}\right] \beta_{1} \boldsymbol{x}-\boldsymbol{x}^{\prime} \beta_{1}\left[\widehat{P}_{1}-P_{0}\right] \beta_{1} \boldsymbol{x}\right] \\
& =\min _{\hat{x} \in \mathbb{R}^{n}} E\left[\boldsymbol{x}^{\prime} \beta_{1}\left[P_{1}-\widehat{P}_{1}\right] \beta_{1} \boldsymbol{x}\right] .
\end{aligned}
$$


The first-order condition can be given as (in the expectation below, note that $\hat{x}$ is fixed, since it only depends on the covariates and hyperparameters governing the distribution of $\boldsymbol{x}$ ):

$$
E\left[\beta_{1}^{2}\left(P_{1}-\widehat{P}_{1}\right) \boldsymbol{x}\right]=E\left[\beta_{1}^{2}\left(I-\widehat{P}_{1}\right) \boldsymbol{x}\right]=\beta_{1}^{2}\left(I-\widehat{P}_{1}\right) E[\boldsymbol{x}]=0 .
$$

This says that if $\hat{\boldsymbol{x}}$ is optimal, then the column space of $\hat{\boldsymbol{x}}$ and $Z$ must contain $E[\boldsymbol{x}]$. Thus, one optimal solution is $\hat{\boldsymbol{x}}=E[\boldsymbol{x}]$ : the dosage. This solution may not be unique, just as the basis vectors of a vector space are not unique.

\section{Acknowledgements}

We acknowledge the work of Jennifer James and Nathaniel Bowerman in early phases of this project. This work was funded by the National Human Genome Research Institute (R15HG004543; R15HG006915). We acknowledge the use of the Hope College parallel computing cluster for assistance in data simulation and analysis.

\section{References}

-1 International HapMap 3 Consortium, Altshuler DM, Gibbs RA, Peltonen L, et al: Integrating common and rare genetic variation in diverse human populations. Nature 2010;467: 52-58.

-2 1000 Genomes Project Consortium, Abecasis GR, Altshuler D, Auton A, et al: A map of human genome variation from population-scale sequencing. Nature 2010;467:1061-1073.

$>3$ Sullivan PF, de Geus EJC, Willemsen G, et al: Genome-wide association for major depressive disorder: a possible role for the presynaptic protein Piccolo. Mol Psychiatry 2009;14: 359-375.

4 Barrett JC, Hansoul S, Nicolae DL, et al: Genome-wide association defines more than 30 distinct susceptibility loci for Crohn's disease. Nat Genet 2008;40:955-962.
Zabaleta J, Su LJ, Lin HY, Sierra RA, Hall MC, Sartor AO, Clark PE, Hu JJ, Ochoa AC: Cytokine genetic polymorphisms and prostate cancer aggressiveness. Carcinogenesis 2009; 30:1358-1362.

-6 Li Y, Willer CJ, Ding J, Scheet P, Abecasis GR: $\mathrm{MaCH}$ : using sequence and genotype data to estimate haplotypes and unobserved genotypes. Genet Epidemiol 2010;34:816-834.

7 Howie BN, Donnelly P, Marchini J: A flexible and accurate genotype imputation method for the next generation of genome-wide association studies. PLoS Genet 2009; 5:e1000529.

8 Lin DY, Hu Y, Huang BE: Simple and efficient analysis of disease association with missing genotype data. Am J Hum Genet 2008;82: 444-452.

$>9 \mathrm{Hu}$ YJ, Lin DY: Analysis of untyped SNPs: maximum likelihood and imputation methods. Genet Epidemiol 2010;34:803-815.
10 Zheng J, Li Y, Abecasis GR, Scheet P: A comparison of approaches to account for uncertainty in analysis of imputed genotypes. Genet Epidemiol 2011;35:102-110.

11 Freidlin B, Zheng G, Li Z, Gastwirth JL: Trend tests for case-control studies of genetic markers: power, sample size and robustness. Hum Hered 2002;53:146-152.

12 Wu MC, Lee S, Cai T, Li Y, Boehnke M, Lin $\mathrm{X}$ : Rare-variant association testing for sequence data with the sequence kernel association test. Am J Hum Genet 2011;89:82-93.

13 Lang B: Direct solvers for symmetric eigenvalue problems; in Grotendorst J (ed): Modern Methods and Algorithms of Quantum Chemistry. Julich: John von Neumann Institute for Computing, 2000, pp 231-259. 